\title{
Spontaneous Self-Organisation Of Gold Nanoparticles Into Ordered Two- Dimensional Arrays
}

\author{
C.J.Kiely", L.S.Cheung ${ }^{*}$ and M.Brust ${ }^{*}$, \\ \# Department of Materials Science and Engineering, Lehigh University, PA 10815, USA \\ * Center for Nanoscale Science, University of Liverpool, Merseyside, L69 3BX, UK.
}

Since the realization that nanoscale metal and semiconductor particles exhibit unusual size-dependant properties, there has been great interest in using these particles as building blocks for new materials with novel characteristics. One of the most straightforward ways for constructing ordered superstructures from such particles is self-assembly, whereby a colloidal solution of chemically stabilized nanoparticles is placed on a substrate and the solvent is allowed to evaporate in a controlled fashion. For particles with a narrow monomodal size distribution, regular hexagonal close packed monolayer rafts are usually generated [1]. More recently, we have shown that ligand-stabilised metal nanoparticle preparations can spontaneously organize themselves into complex two-dimensional arrays [2,3]. Based on the geometrical packing of hard spheres, a clear parallelism was demonstrated between the bimodal nanoparticle structures formed and those predicted for intermetallic alloys on the atomic scale and colloidal crystals on the micron scale. In this paper, we extend these studies by analyzing the superlattice structures formed when two different sized monomodal nanoparticle preparations are mixed in controlled proportions and then deposited onto a substrate.

Two stock toluene solutions containing alkane-thiol stabilized $\mathrm{Au}$ nanoparticles were prepared following the two-phase method of Brust et al.[4]. The measured metallic core sizes in these two separate nanoparticle preparations were $5 \pm 1 \mathrm{~nm}$ and $2 \pm 1 \mathrm{~nm}$ respectively. These stock solutions were then systematically mixed in varying proportions to obtain solutions with large-to-small particle ratios of 1:2, 1:15 and 1:100. These mixtures were subsequently pipetted onto continuous carbon thin films, the solvent allowed to evaporate, and then the resultant nanoparticle arrays were examined by TEM.

For the 1:2 large-to-small mixture, the larger particles formed extended hexagonal rafts, around the periphery of which, the smaller particles were found to segregate. In the 1:100 sample, the smaller particles formed a sea of hexagonal and square packed rafts, in which were embedded nuclei of hexagonal rafts of the larger particles. By far the most interesting structures were observed in the sample containing a 1:15 ratio of 5-to-2nm particles. As well as segregated regions of 5nm hexagonal rafts and $2 \mathrm{~nm}$ hexagonal rafts, there were numerous regions where the two particle types had intimately mixed to form ordered superlattice structures. Fig.1 shows a region where the larger (Atype) particles form a regular hexagonal raft and the smaller (B-type) particles sit in each of the trigonal interstices. The local number ratio of large to small particles in this raft is $1: 2$ and so it is designated as an $\mathrm{AB}_{2}$ structure. This $\mathrm{AB}_{2}$ type of nanoparticle superlattice has been observed previously [2] and is isostructural with $\mathrm{AlB}_{2}$. Fig.2 shows a completely new of type of ordering whereby the large particles form an extended square raft; each $5 \mathrm{~nm}$ particle is then symmetrically surrounded by eight $2 \mathrm{~nm}$ particles. The local stiochiometry of this structure is $\mathrm{AB}_{4}$, and as far as we are aware it has no bulk intermetallic analogue. A third type of ordering observed is shown in Fig. 3 where, in projection at least, it appears that we have alternating parallel rows of large and small particles. To date, it has not possible to experimentally determine the $A: B$ ratio of this new alternating line structure, but from modeling studies we estimate it to be about $\mathrm{A}_{2} \mathrm{~B}_{9}$. The origins and co-existence 
of all these superlattice structures will be explained in terms of sequential stacking of alternating rafts of large and small particles having a variety of different geometries and stacking sequences.

Another fascinating phenomenon found in the 1:15 sample is shown in Fig.4, whereby a hexagonal raft of larger nanoparticles forms an epitaxial interface with an $\mathrm{AB}(c . f . \mathrm{NaCl})$ superlattice structure. These $\mathrm{AB}$ structures were frequently found intercalated within the larger hexagonal rafts. It is clear that although these structures are essentially only one or two layers thick, the ribbon of $\mathrm{AB}$ material is pseudomorphically strained by $\sim 8 \%$ into a tetragonal lattice. To our knowledge, this is the first observation of an epitaxial interface structure in these nanoparticle systems.

[1] Murray CB, Kagan CR and Bawendi MG, Science, 270, (1995), 1335.

[2] Kiely CJ, Fink J, Brust M, Bethell D and Schiffrin DJ, Nature, 396, (1998), 444.

[3] Kiely CJ, Fink J, Zheng JG, Brust M, Bethell D and Schiffrin DJ, Adv. Mats., 12, (2000), 640.

[4] Brust M, Walker M, Bethell D, Schiffrin R and Whyman R, J.Chem.Soc.Comm., (1994), 801.

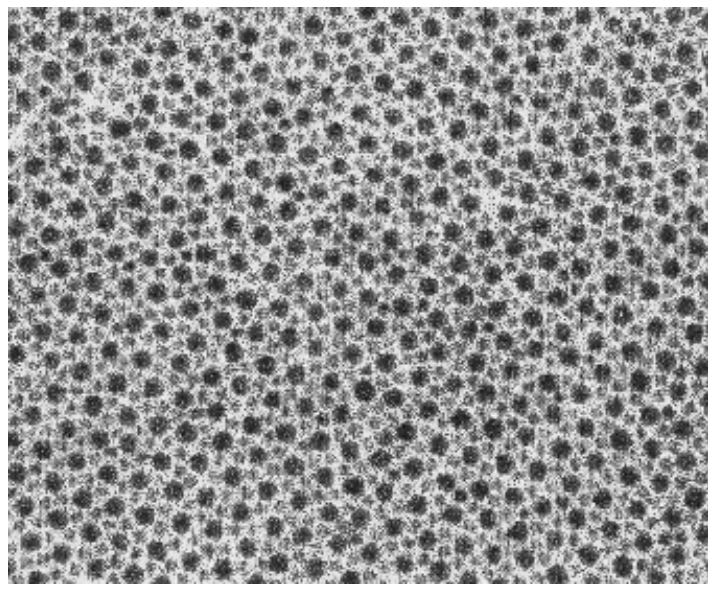

Figure $1 \mathrm{AB}_{2}$ superlattice

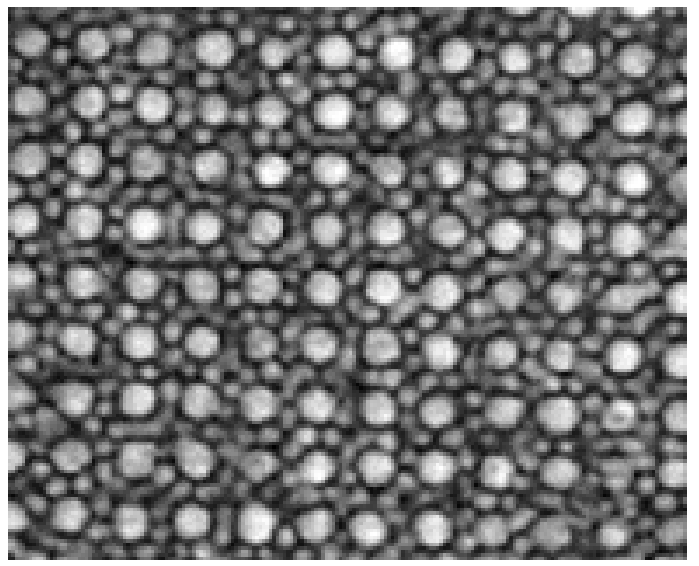

Figure $2 \mathrm{AB}_{4}$ superlattice

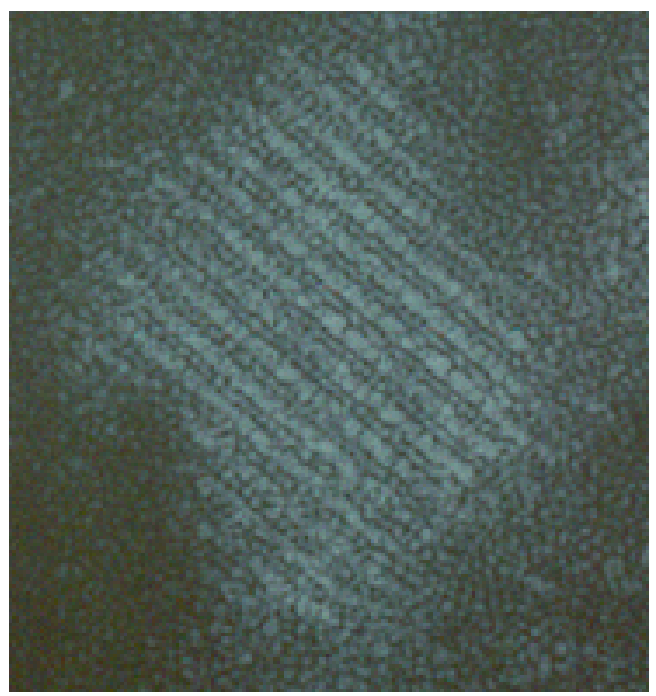

Figure $3 \mathrm{~A}_{2} \mathrm{~B}_{9}$ superlattice

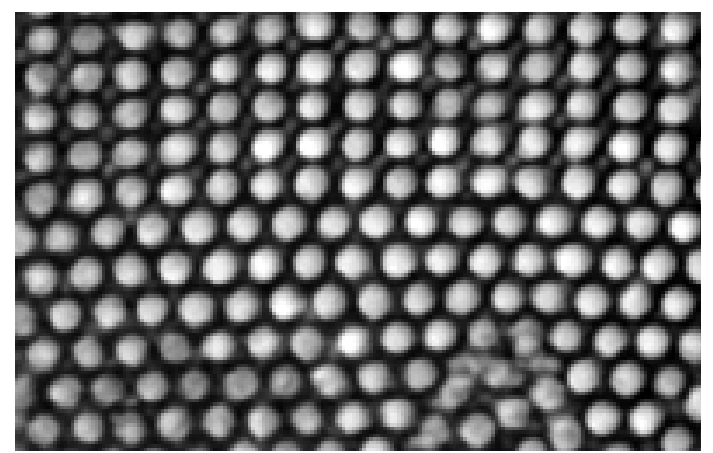

Figure 4 Interface between hexagonal A raft and $A B$ superlattice 